\title{
Selective Transvenous Embolization Original Combined with Balloon Sinoplasty for the Treatment of Intracranial Dural Arteriovenous Fistulas with Sinus Occlusion
}

Yoshiko Sagara, ${ }^{1}$ Hiro Kiyosue, ${ }^{1}$ Shuichi Tanoue, ${ }^{1}$ Yuzo Hori, ${ }^{2}$ Mika Okahara, ${ }^{1}$ Takeaki Dotsu, ${ }^{1}$ Satomi Ide, ${ }^{2}$ and Hiromu Mori ${ }^{1}$

Purpose: Transvenous sinus packing with coils has been widely accepted as a curative treatment method for dural arteriovenous fistulas (DAVFs) with sinus occlusion. Some technical reports have described using luminal angioplasty or stent placement to reconstruct antegrade venous drainage. In addition, recent anatomical considerations of the parasinuses have enabled us to achieve selective embolization of the shunted venous pouch. Here, we report the technical results from five cases of DAVF with sinus occlusion treated by selective transvenous embolization combined with balloon sinoplasty (STVEBS).

Materials and Methods: Five consecutive patients who underwent STVEBS between March 2009 and March 2015 in our institution were retrospectively reviewed. All of the patients were males and were between 68 and 83 years of age. Three patients had a DAVF at the transverse sinus (TS) with ipsilateral sigmoid sinus (SS) or jugular vein (JV) occlusion. One patient had concurrent DAVFs at the right SS and left TS with left transverse sigmoid sinus (TSS) occlusion and a history of right JV ligation. The last patient had an isolated DAVF at the superior sagittal sinus.

Results: In three of the five cases, the fistula was completely obliterated by selective embolization, and antegrade sinus flow was successfully reconstructed by sinoplasty. The remaining two patients showed recanalization of DAVFs at the occluded sinus, and the sinus reopened after balloon angioplasty. These patients were subsequently treated with sinus packing. In all five patients, angiography showed complete obliteration of the DAVF. No complications occurred, and clinical symptoms improved. No recurrence was observed in any patient during the follow-up period, which ranged from 7 to 79 months, and the reconstructed sinus was patent at the last follow-up in all three patients who had undergone successful STVEBS.

Conclusion: STVEBS can obliterate DAVFs with re-establishing the antegrade sinus flow and would be an effective and safe treatment method for cases of DAVF with sinus occlusion.

Keywords > dural arteriovenous fistula, sinus occlusion, angioplasty, transvenous embolization

$\overline{{ }^{1} \text { Department of Radiology, Oita University Faculty of Medicine, }}$ Yufu, Japan

${ }^{2}$ Department of Radiology, Nagatomi Neurosurgical Hospital, Oita, Japan

Received: April 22, 2016; Accepted: August 11, 2016

Corresponding author: Yoshiko Sagara. Department of Radiology, Oita University Faculty of Medicine, Idaigaoka, Hasama, Yufu, Oita 879-5593, Japan

Email: ysagara@oita-u.ac.jp

(C)2016 The Editorial Committee of Journal of Neuroendovascular

Therapy. All rights reserved.

\section{Introduction}

Transvenous sinus packing with coils has been widely accepted as a curative treatment method for dural arteriovenous fistulas (DAVFs) with sinus occlusion. ${ }^{1-3)}$ Some technical reports have described using luminal angioplasty or stent placement to treat occluded dural sinuses and reconstruct antegrade venous drainage. ${ }^{4-16)}$ In addition, recent anatomical considerations of the parasinuses have enabled us to achieve selective embolization of the shunted 
venous pouch. ${ }^{17)}$ To our knowledge, selective transvenous embolization (TVE) combined with balloon sinoplasty (STVEBS) has not been reported. Here, we report the technical results in five cases of DAVF with sinus occlusion that were treated by STVEBS.

\section{Materials and Methods}

\section{Patients}

Five consecutive patients who underwent STVEBS between March 2009 and March 2015 in our institution were retrospectively reviewed. STVEBS were performed for isolated DAVF or DAVFs with shunted venous pouch in which selective embolization can be achieved, also with occlusion of antegrade sinus drainage. All of the patients were males and were between 68 and 83 years of age. The clinical characteristics of the five patients are summarized in Table 1. Three patients had a DAVF at the transverse sinus (TS) with ipsilateral sigmoid sinus (SS) or jugular vein (JV) occlusion. One patient had concurrent DAVFs at the right SS and left TS with left TSS occlusion and a history of right JV ligation. The last patient had an isolated DAVF at the superior sagittal sinus (SSS). Two patients had cerebral or cerebellar hemorrhages, one patient had dementia, and two patients were asymptomatic.

Institutional review board approval is not required for retrospective studies at our institution.

\section{Endovascular procedure}

Each patient provided written informed consent for angiography and interventional procedures before being examined. All procedures were performed under local anesthesia using biplane angiography equipment. Basically, TVE was performed using a triple coaxial catheter technique. A 4-5F guiding sheath/catheter system was advanced into the JV via a bilateral femoral venous approach, and the $2.7 \mathrm{~F}$ high flow or $4 \mathrm{~F}$ supporting catheter/microcatheter was then inserted into the affected parasinus shunted pouch with a 0.010- or 0.014-inch microguidewire. Subsequently, the shunted pouch was occluded with detachable coils. Angioplasty was performed with a 2.5- to 5-mm balloon catheter at nominal pressure for 20 to 60 seconds (Gateway, Boston Scientific, Natick, MA, USA; Siden Kaneka, Osaka, Japan; Attendant, Terumo Clinical supply, Gifu, Japan) positioned in the occluded sinus and serially inflated along the sinus from the distal to the proximal portion. In our institution, TVE is the first-line treatment option for sinus-type DAVF, because transarterial embolization (TAE) has some risks

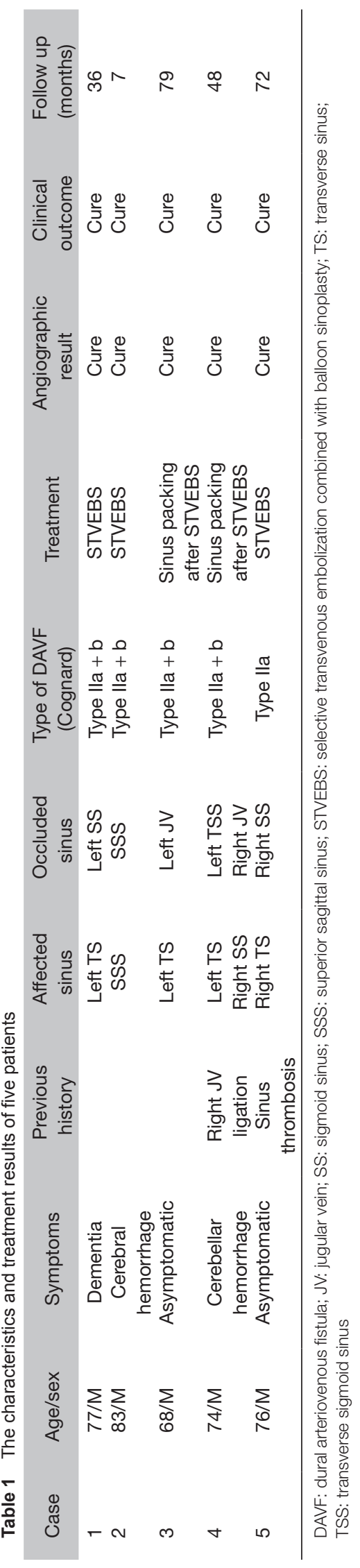

Journal of Neuroendovascular Therapy Vol. 10, No. 5 (2016) 
including migration of embolic material into the dural sinus or dangerous anastomosis and cranial nerve injury.

\section{Results}

The treatment results are summarized in Table 1. In three of the five cases (Cases 1, 2, and 5), the fistula was completely obliterated by selective embolization, and antegrade sinus flow was successfully reconstructed by sinoplasty (Figs. 1 and 2). Among these three patients, one case with isolated DAVF at the SSS (Case 2) first underwent sinoplasty to improve the congestive venous flow, and selective TVE was performed 18 days later. The other patients underwent STVEBS during the same session. Three cases showed recanalization of DAVFs with reopening of the sinus by balloon angioplasty. In one case (Case 1), the shunted sinus was treated by additional selective TVE, and the other two cases (Cases 3 and 4) were subsequently treated with sinus packing. In all the five patients, angiography showed complete obliteration of the DAVF immediately after treatment. No complications occurred during the procedures, and clinical symptoms improved. No recurrence was observed in any patient during the follow-up period, which ranged from 7 to 79 months, and the reconstructed sinus was patent at the last follow-up in all three patients who had undergone successful STVEBS.

\section{Illustrative Cases}

\section{Case 1}

A 77-year-old male presented with progressive dementia. A DAVF at left TSS was suspected on Magnetic resonance (MR) examination. MRI FLAIR image shows cortical venous congestion (Fig. 1a). External carotid angiography (ECAG) revealed that the left TS DAVF was mainly fed by the bilateral occipital artery (OA) and drained into the contralateral TS with retrograde drainage into the SSS (occlusion of distal portion) and Straight sinus (StS) with marked cortical venous reflux. The venous flow at the left TS-SS was not observed (Figs. 1b and 1c). TVE was performed for the treatment of DAVF. A microcatheter was navigated into the parasinus at the left TS via a bilateral femoral venous approach using a triple coaxial system (5 Fr. VTA catheter, sniper high flow, Terumo clinical supply; 1.9F Carnelian Marvel, Tokai Medical Products, Aichi, Japan; 4 Fr. Cerulean, Medikit Tokyo, Japan; Excelsior 1018 Stryker, Kalamazoo, MI, USA) (Fig. 1d), and selective embolization of the shunted pouch was obtained with detachable coils (Trufill Orbit Galaxy, Codman Neuroendovascular, Johnson \& Johnson, Miami, FL, USA; ED coil, Inifini, Kaneka Osaka, Japan; Target coil, Stryker neurovascular, Fremont, CA, USA) (Fig. 1e). Frontal views of left common carotid angiography (CCAG) after TVE reveals the disappearance of the DAVF but still shows a congestive cerebral vein (Figs. 1f and 1g). Therefore, sinoplasty was performed using 3-mm/15-mm monorail balloon catheters (arrows, Gateway monorail, Stryker) with 5-6 atm/20-30 sec to restore antegrade drainage along the TSS eight times in a row (Fig. 1h). Lateral view of left CCAG immediately after sinoplasty reveals a reopening of the DAVF at the occluded TSS with reopening of the sinus lumen (Fig. 1i). Additional selective TVE with coils at the reopened shunted pouch was obtained (Fig. 1j). Frontal (Figs. 1k and 1I) and lateral (Fig. 1m) view of follow-up angiograms 11 days after STVEBS shows a disappearance of the DAVF with recanalization of the TSS, which received the cerebral flow from the vein of Labbe and mainly drained into the emissary vein. FLAIR image, 3 months after the STVEBS, revealed marked improvement in cortical venous congestion (Fig. 1n). Sinus patency on MR images is maintained without recurrence of DAVF during 3 years follow-up period.

\section{Case 5}

A 75-year-old male presented with right TS DAVF (Cognard type IIa). After 13 months of treatment for the sinus thrombosis at bilateral TS, confluence, SSS, and straight sinus by intravenous administration of heparin, a DAVF at right TS was suspected on MR examination. Right ECAG (Fig. 2a) shows the DAVF at TS with occlusion at proximal SS without cortical venous reflux (Cognard type IIa). Selective TVE was performed for shunted venous pouch (arrows in Fig. 2a) with detachable coils (Fig. 2b), and a disappearance of DAVF was achieved. Subsequently, sinuplasty was performed four times along TSS using balloon catheter (Gateway $2.5 \mathrm{~mm} / 9 \mathrm{~mm}$, Stryker, $6 \mathrm{~atm}$ for 20-30 sec). CCAG before (Fig. 2c) and after (Fig. 2d) the STVEBS presents complete restoration of antegrade venous drainage (arrows) toward the JV.

\section{Discussion}

For cases of DAVFs, particularly those involving the TS, $\mathrm{SS}$, or SSS, it is important to evaluate the drainage pattern of the DAVF and the drainage route of normal cerebral venous flow, because they are strongly related to both clinical symptoms and the treatment strategy. For cases of 
(a)

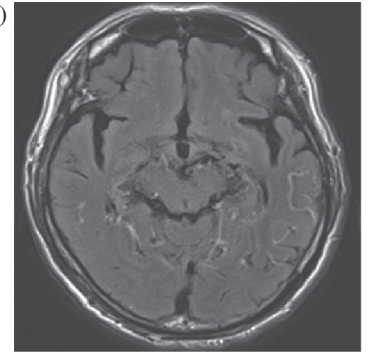

(e)

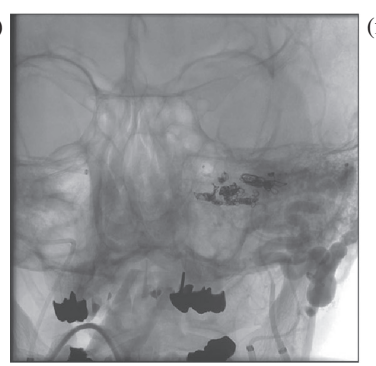

(i)

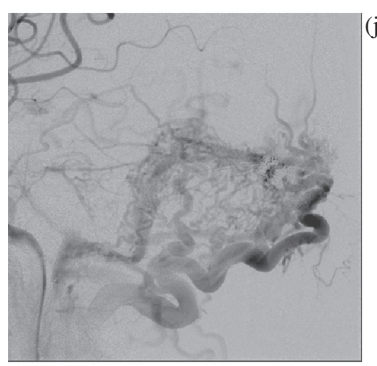

(b)

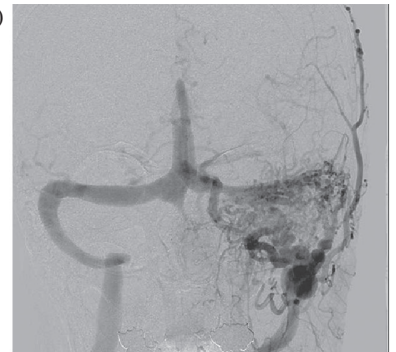

(f)

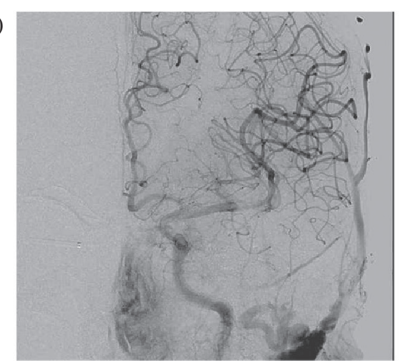

(j)

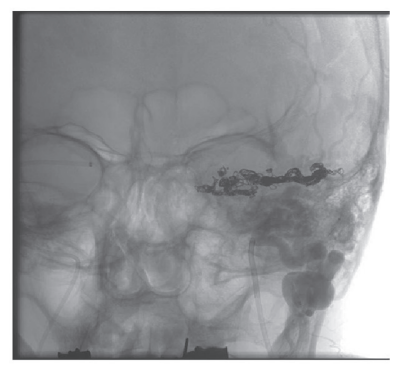

c)

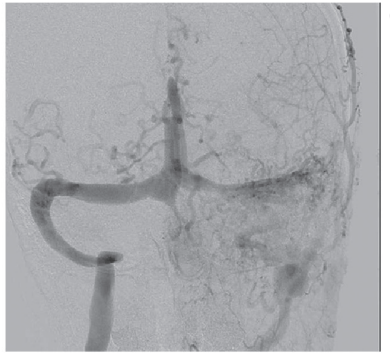

(g)

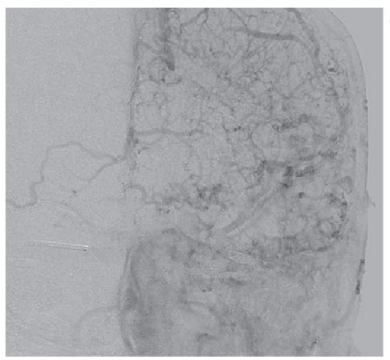

(k)

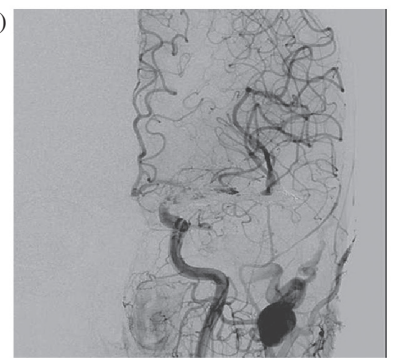

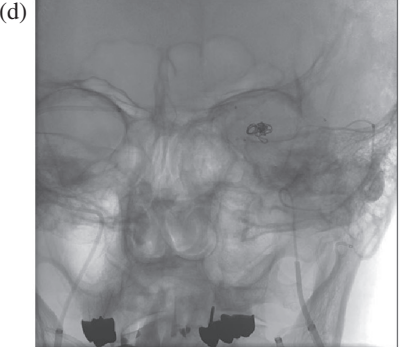

(h)

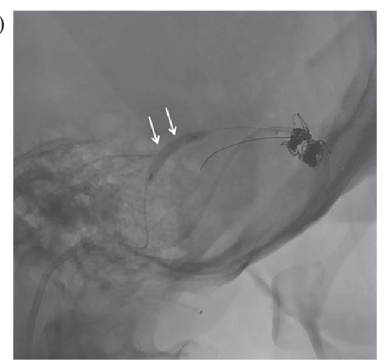

(1)

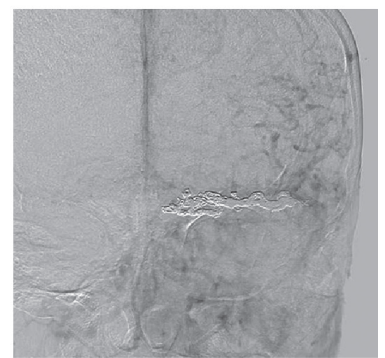

(m)
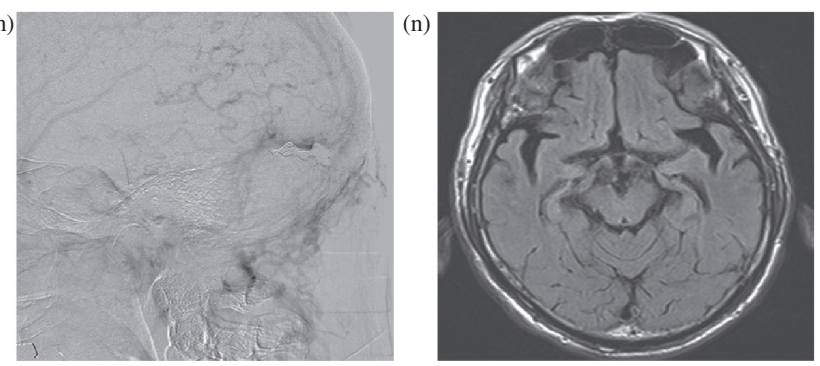

Fig. 1 (Case 1) A 77-year-old male presented with progressive dementia. (a) MRI FLAIR image shows cortical venous congestion. (b, c) The left TS DAVF is mainly fed by the bilateral OA and drains into the contralateral TS with retrograde drainage into the SSS (occlusion of distal portion) and StS with marked cortical venous reflux on frontal view of ECAG. The venous flow at the left TS-SS is not observed. (d) Venography shows a microcatheter navigated into the parasinus at the left TS via a bilateral femoral venous approach using a triple coaxial system (5 Fr. VTA catheter, sniper high flow, Terumo clinical supply, Gifu; 1.9F Carnelian Marvel, Tokai Medical Products, Aichi; 4 Fr. Cerulean, Medikit Tokyo; Excelsior 1018 Stryker, Kalamazoo, MI, USA) (e) Selective embolization of the shunted pouch is obtained with detachable coils (Trufill Orbit Galaxy, Codman Neuroendovascular, Johnson \& Johnson, Miami, FL, USA; ED coil, Inifini, Kaneka Osaka, Japan; Target coil, Stryker neurovascular, Fremont, CA,USA). (f, g) Frontal views of left CCAG after TVE reveal the disappearance of the DAVF but still show a congestive cerebral vein. (h) Lateral view of fluorogram during sinoplasty using 3-mm/15-mm monorail balloon catheters (arrows, Gateway monorail, Stryker) with 5-6 atm/20-30 sec to restore antegrade drainage along the TSS eight times in a row. (i) Lateral view of left CCAG immediately after sinoplasty reveals a reopening of the DAVF at the occluded TSS with reopening of the sinus lumen. (j) AP view of a fluorogram after additional TVE with coils at the reopened shunted pouch. (k, I) Frontal and $(\mathbf{m})$ lateral view of follow-up angiograms 11 days after STVEBS shows a disappearance of the DAVF with recanalization of the TSS, which received the cerebral flow from the vein of Labbe and mainly drained into the emissary vein. (n) FLAIR image, 3 months after the STVEBS, reveals marked improvement in cortical venous congestion. AP: anteroposterior; CCAG: common carotid angiography; DAVF: dural arteriovenous fistula; ECAG: external carotid angiography; SS: sigmoid sinus; STVEBS: selective transvenous embolization combined with balloon sinoplasty; StS: straight sinus; TS: transverse sinus; TSS: transverse sigmoid sinus; TVE: transvenous embolization 
(a)

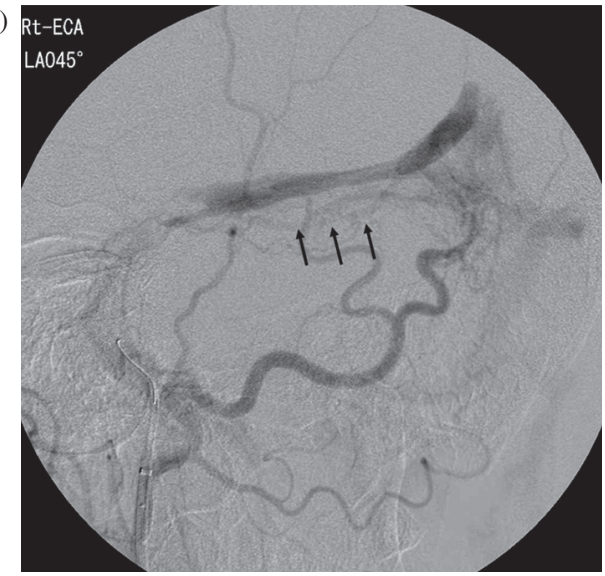

(c)

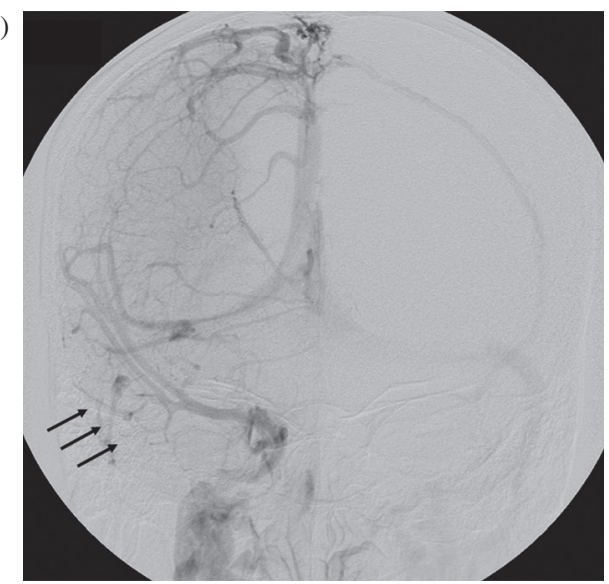

(b)

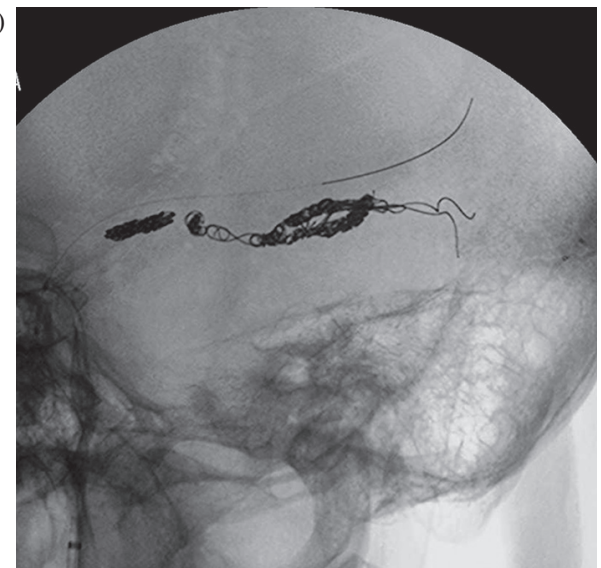

(d)

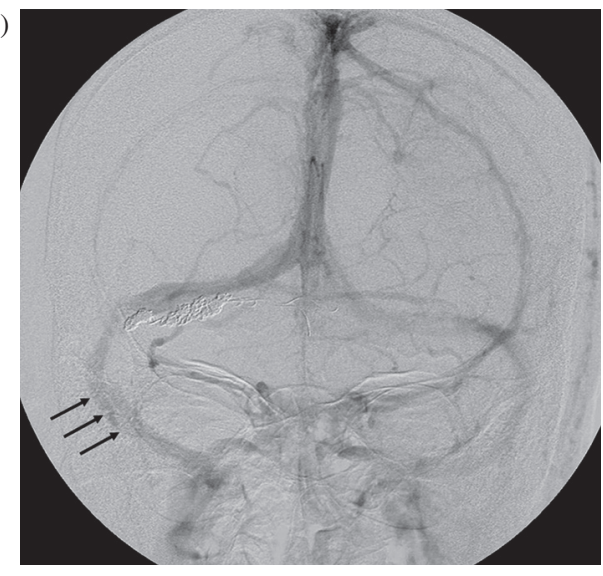

Fig. 2 (Case 5) A 75-year-old male with right transverse sinus DAVF (Cognard type Ila). (a) Right ECAG shows a DAVF at transverse sinus with occlusion at proximal sigmoid sinus without cortical venous reflux (Cognard type Ila). Selective TVE was performed for shunted venous pouch (arrows) with detachable coils (b) and a disappearance of DAVF was achieved. Subsequently, sinuplasty was performed four times along transverse-sigmoid sinus using balloon catheter (Gateway $2.5 \mathrm{~mm} / 9 \mathrm{~mm}$, Stryker, 6 atm for 20-30 sec). CCAG before (c) and after (d) the STVEBS presents complete restoration of antegrade venous drainage (arrows) toward the jugular vein. CCAG: common carotid angiography; DAVF: dural arteriovenous fistula; ECAG: external carotid angiography; STVEBS: selective transvenous embolization combined with balloon sinoplasty; TVE: transvenous embolization

DAVF with sinus occlusion, transvenous sinus packing with coils has been widely accepted as a treatment method. However, packing occluded sinus, which is necessary for the cure of DAVFs, can also lead to severe complications, such as intracranial hemorrhage, due to the occlusion of normal cerebral venous flow draining into the fistulous sinus. If the DAVF-affected sinus receives normal cerebral venous drainage, it cannot be sacrificed. Therefore, preserving or re-establishing cerebral venous outflow can be an ideal treatment method.

Several previous reports have indicated that angioplasty (recanalization) for occluded sinus/venous stenosis using stent placement and balloon angioplasty is a good treatment option for the reconstruction of antegrade sinus drainage. Dural venous stent insertion has been performed for the initial treatment of a stenotic or thrombosed dural sinus that is causing intracranial venous hypertension. Since 1996, stent placement has been used as a conservative treatment method for DAVF.4) Malek et al..$^{5}$ presented a mechanical recanalization using balloon angioplasty and stent deployment in the thrombosed occipital sinus to provide venous outflow for the treatment of venous hypertension after the embolization of multiple DAVFs. In 2000, Murphy et al. ${ }^{6}$ reported on a case of a type IV TS-SS DAVF treated with recanalization using transluminal angioplasty and coronary stent placement in the partially thrombosed fistulous sinus. Complete obliteration of the DAVF was achieved with recanalization of the normal cerebral venous outflow pathways. Levrier et al. ${ }^{11)}$ reported on 10 cases of TS or SS DAVF that were cured or significantly improved after inserting a sinus self-expanding stent, and an angiogram immediately after the procedure 
revealed a significant decrease $(40 \%)$ or occlusion $(40 \%)$ of shunt flow. Previous reports on balloon angioplasty/ stenting to restore venous outflow in cases of DAVF are summarized in Table 2. Three studies reported restored sinus outflow, which drains from the DAVF at different sinus levels, ${ }^{5,7,10)}$ and the remaining studies evaluated sinuplasty within the DAVF-affected sinus. , $^{6,8,9,11-16)}$

Some authors have emphasized that stent placement for the treatment of DAVFs is a promising technique, because stent placement in the DAVF-affected sinus and angioplasty could compress the vessels in the sinus wall and occlude the arteriovenous shunt flow hemodynamically. In addition, recanalization of the sinus could restore venous outflow and correct venous hypertension. However, neither the long-term effects nor the complications of stent placement for sinus occlusion are known. To prevent restenosis or thrombosis, antithrombotic and/or antiplatelet therapy is required. In addition, complete obliteration of the fistulous sinus with stents alone remains challenging and it is not always efficient in treating DAVF and may require complementary treatments, such as TVE, TAE, or radiosurgery. Stent placement alone may carry a risk of a residual shunt with cortical venous reflux, which may cause more aggressive symptoms.

With respect to TVE for the treatment of DAVFs, recent anatomical considerations of the parasinuses have enabled us to achieve selective embolization of the shunted venous pouch. ${ }^{17-19)}$ Selective TVE of the shunted pouch is a useful technique, particularly for the treatment of DAVFs without cortical venous reflux (Cognard type I and IIa), which can occlude the shunt while preserving sinus drainage. Even in the treatment of DAVFs with cortical venous reflux (Cognard type IIb and IIa + b), selective embolization could initially reduce the shunting flow and achieve safer and more effective treatment results than could simple sinus packing. In 4 of the 10 cases reported by Levrier et al., ${ }^{11)}$ after stent placement into the sinus for the treatment of TSS DAVF, the remnant shunt cavity between the external dural wall of the sinus and the stent wall was embolized using platinum coils and advancing the microcatheter through the stent cell. They occluded the residual shunt within the sinus after reducing the shunted flow by stenting, which differs from selective embolization of the shunted venous pouch. To our knowledge, no previous reports have described selective embolization of the shunted venous pouch in combination with sinus recanalization.

We treated five cases of DAVF with sinus occlusion using STVEBS. One case involved an isolated DAVF at the SSS.

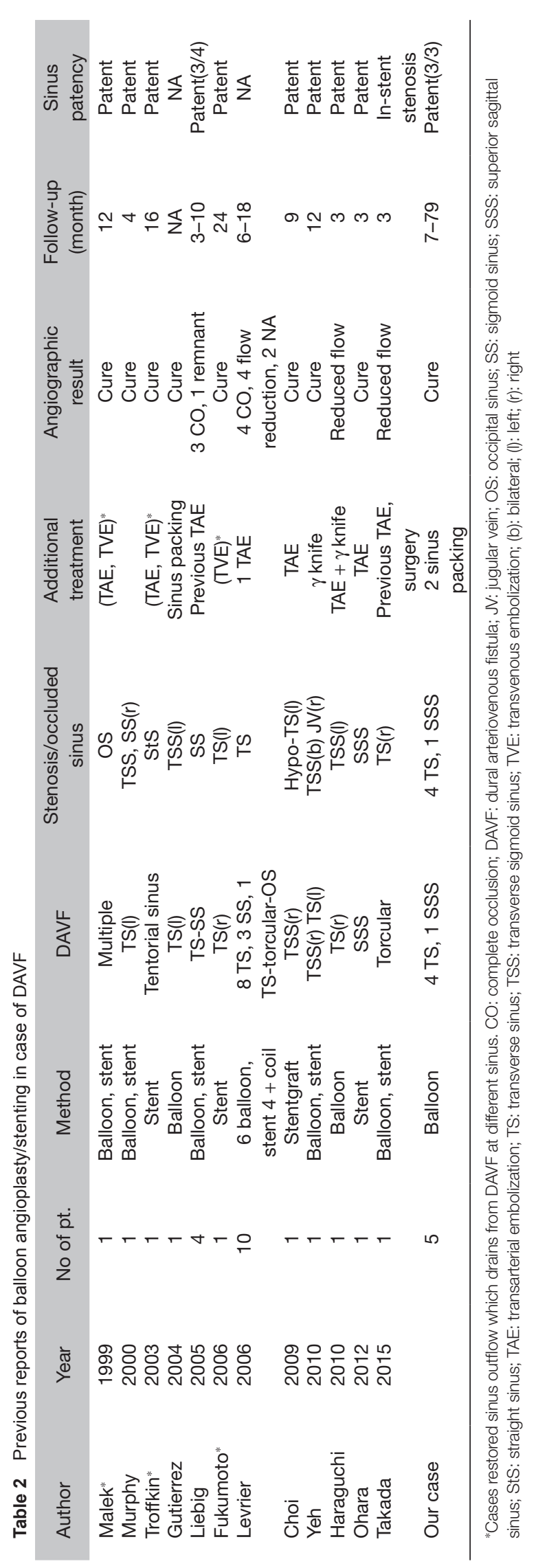

Journal of Neuroendovascular Therapy Vol. 10, No. 5 (2016) 
The remaining four cases were initially treated with selective TVE, and after the shunted venous flow was reduced, balloon angioplasty was performed to restore venous outflow. In three of the five cases, the fistula was completely obliterated while preserving antegrade sinus flow. The remaining two cases showed recanalization of DAVFs at the occluded sinus after balloon sinoplasty. These patients were subsequently treated with sinus packing. Therefore, all five patients achieved complete obliteration of the DAVF without complications, and no recurrence was observed during the follow-up period, which ranged from 7 to 79 months. STVEBS can obliterate DAVFs while re-establishing antegrade sinus flow and would be an effective and safe treatment method for cases of DAVFs with sinus occlusion.

Gutierrez et al. ${ }^{8)}$ reported on a case of type IIa DAVF at the TS with ipsilateral sinus stenosis, in which an unexpected worsening of the retrograde flow into the cortical vein developed (conversion to Cognard type IIa $+\mathrm{IIb}$ ) after balloon angioplasty of the stenotic sinus. Sinus packing was performed to occlude the DAVF. In three of our five cases, the shunted sinus, which was obliterated by selective TVE, reopened after angioplasty and required additional selective TVE $(n=1)$ and sinus packing $(n=2)$. We suspect the reason of recanalization of a DAVF after sinoplasty that shunt flow is temporarily occluded with thrombi produced by occlusion of venous pouch and after sinuplasty, a migration or decrease in thrombi may cause recanalization of the fistula. Therefore, not all cases of DAVF can be managed by STVEBS. Other challenges, including a longer procedure time, higher costs, and greater radiation exposure, still need to be addressed. We also cannot refer to the change of venous pressure, because we did not measure venous pressure before and after the sinoplasty.

\section{Conclusion}

STVEBS can obliterate DAVFs and re-establish antegrade sinus flow and would be an effective and safe treatment method for cases of DAVFs with sinus occlusion.

\section{Disclosure Statement}

The authors declare no conflicts of interest.

\section{References}

1) Kiyosue H, Hori Y, Okahara M, et al: Treatment of intracranial dural arteriovenous fistulas: current strategies based on location and hemodynamics, and alternative techniques of transcatheter embolization. Radiographics 2004; 24 : 1637-1653.

2) Naito I, Iwai T, Shimaguchi H, et al: Percutaneous transvenous embolisation through the occluded sinus for transversesigmoid dural arteriovenous fistulas with sinus occlusion. Neuroradiology 2001; 43: 672-676.

3) Komiyama M, Ishiguro T, Matsusaka $Y$, et al: Transfemoral, transvenous embolisation of dural arteriovenous fistula involving the isolated transverse-sigmoid sinus from the contralateral side. Acta Neurochir (Wien) 2002; 144: 1041-1046; discussion 1046.

4) Urtasun F, Biondi A, Casaco A, et al: Cerebral dural arteriovenous fistulas: percutaneous transvenous embolization. Radiology 1996; 199: 209-217.

5) Malek AM, Higashida RT, Balousek PA, et al: Endovascular recanalization with balloon angioplasty and stenting of an occluded occipital sinus for treatment of intracranial venous hypertension: technical case report. Neurosurgery 1999; 44: 896-901.

6) Murphy KJ, Gailloud P, Venbrux A, et al: Endovascular treatment of a grade IV transverse sinus dural arteriovenous fistula by sinus recanalization, angioplasty, and stent placement: technical case report. Neurosurgery 2000; 46: 497-500; discussion 500-1.

7) Troffkin NA, Graham CB, Berkmen $T$, et al: Combined transvenous and transarterial embolization of a tentorialincisural dural arteriovenous malformation followed by primary stent placement in the associated stenotic straight sinus. Case report. J Neurosurg 2003; 99: 579-583.

8) Gutierrez A, Do HM, Marks MP: Alteration in the venous drainage of a dural arteriovenous fistula following angioplasty. AJNR Am J Neuroradiol 2004; 25: 1086-1088.

9) Liebig T, Henkes $H$, Brew S, et al: Reconstructive treatment of dural arteriovenous fistulas of the transverse and sigmoid sinus: transvenous angioplasty and stent deployment. Neuroradiology 2005; 47: 543-551.

10) Fukumoto $S$, Ueda $T$, Igase $K$, et al: Stenting procedure for sinus stenosis with transverse-sigmoid dural arteriovenous fistulas. A case report. Interv Neuroradiol 2006; 12: 178-184.

11) Levrier O, Metellus P, Fuentes S, et al: Use of a selfexpanding stent with balloon angioplasty in the treatment of dural arteriovenous fistulas involving the transverse and/or sigmoid sinus: functional and neuroimaging-based outcome in 10 patients. J Neurosurg 2006; 104: 254-263.

12) Choi BJ, Lee $\mathrm{TH}, \mathrm{Kim} \mathrm{CW}$, et al: Reconstructive treatment using a stent graft for a dural arteriovenous fistula of the transverse sinus in the case of hypoplasia of the contralateral venous sinuses: technical case report. Neurosurgery 2009; 65: E994-E996; discussion E996. 
13) Yeh PS, Wu TC, Tzeng WS, et al: Endovascular angioplasty and stent placement in venous hypertension related to dural arteriovenous fistulas and venous sinus thrombosis. Clin Neurol Neurosurg 2010; 112: 167-171.

14) Haraguchi K, Matsumoto $Y$, Kondo R, et al: Percutaneous transluminal sinus angioplasty for transverse sinus-sigmoid sinus stenosis associated with recurrent dural arteriovenous fistula: case report. JNET 2010; 4: 33-39.

15) Ohara N, Toyota S, Kobayashi M, et al: Superior sagittal sinus dural arteriovenous fistulas treated by stent placement for an occluded sinus and transarterial embolization. A case report. Interv Neuroradiol 2012; 18: 333-340.

16) Takada S, Isaka F, Nakakuki T, et al: Torcular dural arteriovenous fistula treated via stent placement and angioplasty in the affected straight and transverse sinuses: case report. J Neurosurg 2015; 122: 1208-1213.

17) Kiyosue H, Tanoue S, Okahara M, et al: Angioarchitecture of transverse-sigmoid sinus dural arteriovenous fistulas: evaluation of shunted pouches by multiplanar reformatted images of rotational angiography. AJNR Am J Neuroradiol 2013; 34: 1612-1620.

18) Mironov A: Selective transvenous embolization of dural fistulas without occlusion of the dural sinus. AJNR Am J Neuroradiol 1998; 19: 389-391.

19) Piske RL, Campos CM, Chaves JB, et al: Dural sinus compartment in dural arteriovenous shunts: a new angioarchitectural feature allowing superselective transvenous dural sinus occlusion treatment. AJNR Am J Neuroradiol 2005; 26: 1715-1722. 